\title{
DVO-Newsletter|Der Osteologe DVO und Informationen der Gesellschaft
}

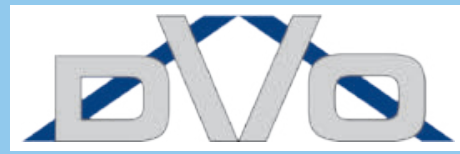

\section{Informationen der Gesellschaft}

\section{Vorstand DVO}

\section{Vorsitzender}

Prof. Dr. Andreas Kurth

Gemeinschaftsklinikum

Mittelrhein gGmbH

Kemperhof Koblenz

Klinik für Orthopädie und Unfallchirurgie, Hand-, Wiederherstellungschirurgie

Koblenzerstr. 115-155

\section{Koblenz}

Tel.: +49(0) 201/3845-617

Fax:+49(0) 201/805-27 17

E-Mail: Kurth@dv-osteologie.de

\section{Vorsitzender}

Prof. Dr. Peyman Hadji

Frankfurter Hormon und

Osteoporosezentrum

Goethestr. 23

60313 Frankfurt am Main

Tel: +49(0) 6921087979

Fax: $+49(0) 6921087978$

E-Mail:p.hadji@outlook.de

\section{Schatzmeister}

Prof. Dr. Peter M. Kern

Klinikum Fulda

Universitätsmedizin Marburg -

Campus Fulda, Medizinische Klinik IV

Pacelliallee 4, 36043 Fulda

Tel.: +49 (0) 661/84-65 81

Fax: +49(0) 661/84-6583

E-Mail: Peter.Kern@klinikum-fulda.de

\section{Sekretär}

Prof. Dr. Christian Meier

Endokrinologische Praxis \& Labor

Osteologisches Universitätsforschungs-

zentrum

Aeschenvorstadt 57 B, 4051 Basel, Schweiz

Tel.: +41 (61) 2649797

Fax+41 (61) 2649796

E-Mail: christian.meier@unibas.ch und

Klinik für Endokrinologie, Diabetologie und Metabolismus, Universitätsspital Basel Petersgraben 4, 4031 Basel, Schweiz

E-Mail: christian.meier@usb.ch

\section{DVO Büro}

Hellweg 92, 45276 Essen

Tel.: +49(0) 201/38 45-617

Fax: +49(0) 201/805-27 17

E-Mail: buero@dv-osteologie.de

\section{DVO im Internet}

http://www.dv-osteologie.org

\section{IMPRESSUM}

Verantwortlich für den Inhalt

Dr. Hermann Schwarz, Freudenstadt
IMPRESSUM

Verantwortlich für den Inhalt

Dr. Hermann Schwarz, Freudenstadt 